\title{
Advances in automated techniques to identify Acinetobacter calcoaceticus-Acinetobacter baumannii complex
}

\author{
Ahmad Ibrahim Bagudo ${ }^{\oplus}$, Godwin Attah Obande ${ }^{\oplus}$, Azian Harun ${ }^{\oplus}$, Kirnpal Kaur Banga Singh ${ }^{*}$
}

\begin{abstract}
Acinetobacter species, particularly those within Acinetobacter calcoaceticus-A. baumannii complex (ACB complex), have emerged as clinically relevant pathogens in hospital environments worldwide. Early and quick detection and identification of Acinetobacter infections is challenging, and traditional culture and biochemical methods may not achieve adequate levels of speciation. Moreover, currently available techniques to identify and differentiate closely related Acinetobacter species are insufficient. The objective of this review is to recapitulate the current evolution in phenotypic and automated techniques used to identify the ACB complex. Compared with other automated or semiautomated systems of bacterial identification, matrix-assisted laser desorption-ionization time-of-flight mass spectrometry (MALDI-TOF MS) demonstrates a high level of Acinetobacter species identification and discrimination, including newly discovered species $A$. seifertii and A. dijkshoorniae.
\end{abstract}

Keywords: Acinetobacter; automation, laboratory; bacterial typing techniques; bacteriological techniques; spectrometry, mass, matrix-assisted laser desorption-ionization

Currently, the genus Acinetobacter comprises about 62 species, 51 with valid species names and 11 unnamed species, most of which are nonclinical isolates, with more species likely to be discovered [1, 2]. Acinetobacter calcoaceticus-Acinetobacter baumannii (ACB) complex is the most worrisome in the genus, being responsible for over $80 \%$ of hospital-acquired infections in immunocompromised patients [3,4]. The ACB complex comprises of Acinetobacter baumannii (genospecies 2), A. calcoaceticus, A. nosocomialis (genospecies 13TU), and A. pittii (genospecies 3) along with the newly identified species, A. seifertii and A. dijkshoorniae [5]. The phenotypic similarity among the members of the ACB complex makes them difficult to distinguish from other species by routine detection methods. Despite their close phenotypic relatedness, there exist differences in their biological characteristics [6], which necessitates accurate identification and differentiation of individual members of the ACB complex [7, 8]. The commonly used techniques used to identify ACB complexes include biochemical tests, gene sequencing (16S rRNA, rpo $\mathrm{B}$ gene) [9], polymerase chain reaction [10, 11], and matrix-assisted laser desorption-ionization time-of-flight mass spectrometry (MALDI-TOF MS) [12]. Despite advances in molecular identification techniques for the ACB complex, the techniques are not suitable for routine diagnosis $[13,14]$ and are applied mainly in research settings [15]. In the present review, we aim to provide an insight into the available

*Correspondence to: Kirnpal Kaur Banga Singh, Department of Medical Microbiology and Parasitology, School of Medical Sciences, Health Campus, Universiti Sains Malaysia, 16150 Kubang Kerian, Kelantan, Malaysia, e-mail: kiren@usm.my

Department of Medical Microbiology and Parasitology, School of Medical Sciences, Health Campus, Universiti Sains Malaysia, 16150 Kubang Kerian, Kelantan, Malaysia

Ә Open Access. $\odot 2020$ Bagudo et al., published by Sciendo. (c) Br-Nc-ND This work is licensed under the Creative Commons Attribution NonCommercial-NoDerivatives 4.0 License. 
phenotypic methods and recent developments in automated systems for rapid and reliable identification of the pathogen species belonging to the ACB complex.

\section{Search methodology}

The information in the present review article was synthesized from Google Scholar, PubMed, and Wiley online library. We explored English-language sources using the following keywords: "Automated or semi-automated identification method of Acinetobacter baumannii complex"; "Biolog system," "BD Phoenix system," "MicroScan WalkAway," "Accelerate Pheno system," "VITEK system," and "MALDITOF MS." Abstracts, theses, or unreviewed work were not included. Emphasis was given to the sources published within the past 7 years.

\section{Phenotypic methods for identification of Acinetobacter species}

Bacterial pathogens are traditionally identified in clinical laboratories based on the morphological and biochemical characteristics displayed on the growth media [16]. Acinetobacter species produce morphological characteristics similar to Enterobacteriaceae [17], with some species of Acinetobacter producing tiny, glowing colonies with no hemolysis on blood agar. However, a few species produce hemolysis on 5\% sheep blood agar. Bouvet and Grimont developed the use of 28 phenotypic tests to identify Acinetobacter species, which focused on the physiological, nutritional, and enzymatic characteristics of Acinetobacter spp. [18]. This method successfully identifies 12 Acinetobacter genospecies, but is unable to identify Acinetobacter iwoffii. Bouvet and Grimont later in 1987 improved their phenotypic tests to comprise growth at temperatures of $37{ }^{\circ} \mathrm{C}, 41^{\circ} \mathrm{C}$, and $44{ }^{\circ} \mathrm{C}$, and in addition included the utilization of 14 carbon sources [17]. Acinetobacter species' ability to grow at different temperatures was used to discriminate $A$. baumannii, A. nosocomialis, A. pittii, and A. calcoaceticus [17, 19]. A further upgrade of the Bouvet and Grimont identification scheme for Acinetobacter species identification to include growth at temperatures of $32{ }^{\circ} \mathrm{C}, 35^{\circ} \mathrm{C}, 41^{\circ} \mathrm{C}$, and $44^{\circ} \mathrm{C}$, and additional biochemical tests such as acid production from glucose, hemolysis, gelatinase production, and utilization of 36 different carbon compounds as energy sources, has improved the scheme [17]. Table 1 shows the biochemical test for the identification of the ACB complex. Although the current phenotypic system appears to be more robust than previously, it still falls short in terms of ease, rapidity, reliability, and consistency. Hence, the emergence of automated systems targeted to solve the challenges of accurate and rapid identification and differentiation of the ACB complex.

\section{Commercial automated systems for the identification of Acinetobacter species}

Recent advances in miniaturization and automation provide promising solutions for rapid bacterial identification and antibiotic susceptibility profiling, which will potentially make a significant impact on the clinical management of infectious diseases [20]. Automated and semiautomated commercial identification systems for the identification of bacteria are in high demand and widely used in clinical microbiology laboratories [21, 22]. Some systems require preisolation of bacteria from the sample and a high concentration of isolates in suspension [23]. Automated bacterial identification systems can measure the differences in protein expression within the bacterial genus or among the bacterial species, providing a particular protein expression with a relatively high degree of accuracy [24]. The commonly used automated or semiautomated bacterial identification systems include the following.

\section{API 20NE system}

The Analytical Profile Index (API) 20NE system is a standardized system for the quick identification of nonfastidious, nonenteric gram-negative rods. It combines 8 conventional tests, 12 assimilation tests, and a database. The API 20NE BioMérieux system demonstrated 92\% accuracy when compared with conventional biochemical tests in the identification of 198 clinical isolates [19, 25]. Bernards et al. [28] achieved $87 \%$ API 20NE accuracy of identification using an updated database (version 5.1). However, the technique was not sufficient to correctly identify and discriminate between Acinetobacter species. Another study that compared API 20NE with 16s rRNA gene sequencing in the identification of gram-negative bacteria, including Acinetobacter, reported successful identification of 58 of 107 isolates. However, some members of the ACB complex could not be accurately identified or discriminated at the species level [26]. 
Table 1. Phenotypic tests for identification of Acinetobacter baumannii (ACB) complex

\begin{tabular}{|c|c|c|c|c|c|c|}
\hline & A. calcoaceticus & A. baumannii & A. pittii & A. nosocomialis & A. seifertii & A. dijkshoorniae \\
\hline $\begin{array}{l}\text { Acid production from } \\
\text { glucose }\end{array}$ & - & + & $V(+)$ & + & + & + \\
\hline \multicolumn{7}{|l|}{ Growth temperature } \\
\hline $37^{\circ} \mathrm{C}$ & $(+)$ & + & + & + & & \\
\hline $41^{\circ} \mathrm{C}$ & $(-)$ & + & V & + & & \\
\hline $44^{\circ} \mathrm{C}$ & - & + & + & v & & \\
\hline \multicolumn{7}{|c|}{ Carbon source utilization test } \\
\hline Citroconitate & - & $V(+)$ & - & - & - & - \\
\hline$\beta$-Alanine & $V(-)$ & + & $V(+)$ & $V(+)$ & $\mathrm{V}(+)$ & + \\
\hline Malate & $\mathrm{V}(-)$ & $\mathrm{V}(+)$ & $V(+)$ & + & $\mathrm{V}(+)$ & \\
\hline Ribose & $V(-)$ & $V(+)$ & $V(-)$ & - & & \\
\hline Leucine & $V(-)$ & $\mathrm{V}(+)$ & $V(+)$ & $V(+)$ & $\mathrm{V}(+)$ & + \\
\hline Ornithine & + & $V(+)$ & $V(+)$ & $V(+)$ & $V(+)$ & $V(+)$ \\
\hline Gentisate & - & $\mathrm{V}(-)$ & $V(-)$ & $V(+)$ & $\mathrm{V}(+)$ & + \\
\hline Azelate & + & $V(+)$ & + & $V(+)$ & & + \\
\hline L-Arabinose & $V(-)$ & $V(+)$ & $\mathrm{V}(+)$ & + & - & + \\
\hline Adipate & + & $\mathrm{V}(+)$ & + & $\mathrm{V}(+)$ & $V(+)$ & + \\
\hline Putrescine & + & & & & & \\
\hline Tartarate & $V(-)$ & $V(-)$ & $V(+)$ & - & $V(+)$ & $V(+)$ \\
\hline Trigonrlline & $\mathrm{V}(-)$ & $V(+)$ & $V(+)$ & $V(-)$ & - & $V(+)$ \\
\hline Sorbinic acid & $(-)$ & $V(+)$ & $(-)$ & $(+)$ & & \\
\hline Glycerate & $V(+)$ & $(-)$ & $V(-)$ & $(-)$ & & \\
\hline Acetylglutamate & $(-)$ & $V(-)$ & $V(-)$ & $V(-)$ & & \\
\hline Asparagine & + & - & - & $(-)$ & & \\
\hline L-Hydroxylproline & $(-)$ & $(+)$ & $(-)$ & $V(-)$ & & \\
\hline Levulinate & $V(-)$ & $\mathrm{V}(-)$ & - & $V(-)$ & & \\
\hline
\end{tabular}

$\mathrm{V}(+)$ variable, majority are positive; $\mathrm{V}(-)$ variable, majority are negative; $(+)$, more than $80 \%$ are positive; $(-), 20 \%$ or less are positive; + , all are positive; -, all are negative; variable means $20 \%-79 \%$ are positive.

\section{Biolog system}

The Biolog system can rapidly identify both gram-positive and gram-negative bacteria. The system works based on universal redox chemistry alone to detect bacterial cellular reactions. By inoculation of microorganism into diverse preselected carbon sources, the system usually produces a specific growth pattern based on the carbon source utilization profile of the bacteria. The resulting metabolic pattern is then compared with the Biolog in-built database to identify the bacteria. The Biolog system works based on the oxidation of 95 substrates from 8 different sets of carbon sources [27]. In a parallel study to compare the Biolog system and DNA-DNA hybridization to identify Acinetobacter species using cluster analysis, Bernards et al. [28] recorded an $84.5 \%$ accurate identification to the genus level by the Biolog system. Being a commercial system, frequent incorporation of new information regarding distinct bacterial strains into the system's database will increase the accuracy of the system in Acinetobacter species identification [28].

\section{BD Phoenix system and MicroScan WalkAway}

The BD Phoenix system works on the redox principle with an appropriate indicator and growth turbidity measurement. While the MicroScan WalkAway system uses a photometer or fluorometer to assess bacterial growth, it is based on fluorescent technology and provides results in an average of $4 \mathrm{~h}$ or $6-42 \mathrm{~h}$ 
with slow-growing bacteria [29]. Synder et al. [30] compared the performance of the BD Phoenix and MicroScan WalkAway systems to identify nonfermentative gram-negative bacilli and reported a $100 \%$ agreement. The MicroScan WalkAway system was reported to have misidentified $A$. baumannii as Ralstonia paucula [31].

\section{VITEK system}

The VITEK system is an entirely automated device that produces quick results typically in less than $7 \mathrm{~h}$, with same-day turnaround time. The system permits ad hoc or group processing of samples and possesses an integrated quality control component with a complete data management system [32]. The VITEK 2 system works on the principles of detection of microbial growth in small wells within plastic cards referred to as gram-negative card BioMérieux. A gram-negative card BioMérieux can identify $A$. baumannii with up to $99 \%$ accuracy, while the phenotypically related $A$. nosocomialis and $A$. pittii are identified as the $A$. baumannii complex. VITEK MS correctly identified A. junnii, A. haemolyticus, and A. jonhsonii [33]. However, VITEK MS failed to distinguish between species within the ACB complex, as members of the ACB complex species are not included on the VITEK MS database, and the substrate used in this system was not specifically designed to identify the Acinetobacter species [17]. VITEK 2 misidentified $4 \mathrm{~A}$. baumannii isolates as Alcaligenes faecalis [31]. A study compared the efficacy of the VITEK 2 anaerobic gram-negative card (ANC) with 16s rRNA identification; the ANC card accurately identified $79.4 \%$ of 301 clinical isolates to genus level, including 100 isolates that were not in the proprietary database. However, the specieslevel identification of those 100 isolates was just 47\% [34].

\section{Accelerate Pheno system}

The Accelerate Pheno system is a fully automated test system that accomplishes identification and antimicrobial susceptibility testing (AST) directly from positive blood cultures within approximately $7 \mathrm{~h}$ [35]. The Accelerate Pheno system is a collection of gel electrofiltration and a large panel of fluorescence in situ hybridization for bacterial identification and morphokinetic cellular analysis, which measures the activities of cells and colonies in the presence of antibiotics over time and employs time-lapse imaging for antimicrobial susceptibility testing. This technique demonstrated excellent sensitivity of $98.6 \%$ and specificity of $99.7 \%$ with the positive and negative predictive values of $100 \%$ for A. baumannii
[36]. In another study involving 101 clinical isolates of Acinetobacter spp., this system recorded a sensitivity of $100 \%$ and $97.6 \%$ for identification of $A$. baumannii and $A$. baumannii complex, respectively, and a specificity of $86.6 \%$ for the A. baumannii complex. The study identified A. baumannii, A. pittii, and $A$. nosocomialis with $100 \%$ sensitivity, although one $A$. radioresistens and one $A$. baylyi were erroneously identified as $A$. baumannii. Commercial automated systems have contributed immensely to the clinical diagnosis of important diseases. However, the weakness of commercial automated methods in terms of accuracy, have been highlighted in some studies [31].

\section{Proteomics-based methods of Acinetobacter species identification}

Molecular methods are not optimal despite strenuous efforts at standardization; it is difficult to compare the results obtained from different laboratories, and the methods are labor-intensive and time-consuming. The search for unique and representative protein biomarkers on intact bacterial cell envelopes to rapidly identify bacteria has been increasing for decades [37, 38]. MALDI-TOF MS is a technique that provides rapid and less cumbersome identification of microorganisms in diagnostic laboratories [39, 40]. MALDI-TOF MS also demonstrates reliability and applicability for the epidemiological typing of bacteria during the outbreak of diseases [41]. It can process up to 384 specimens on a single plate. Its turnaround time is about 5-7 min for each sample, allowing results to be ready within $24 \mathrm{~h}$ of receiving a sample. MALDI-TOF MS requires as low as $10^{4}-10^{6}$ colony forming units (CFU) of cells, and theoretically, identification can be performed using a single colony, obtained in a few hours, from the culture of an infected sample [20].

In a study to optimize MALDI-TOF MS for identification of common bacteria directly from blood samples, of 829 positive blood cultures, MALDI-TOF MS accurately identified 91.5\% as gram-negative bacteria, $88.3 \%$ as gram-positive bacteria, $84.8 \%$ as fungi, $80 \%$ as anaerobic bacteria, and $66.67 \%$ as other rare bacteria, demonstrating that routinely encountered bacteria in positive blood cultures can be identified directly within $1 \mathrm{~h}$ using this method [42]. MALDI-TOF MS has high sensitivity, acceptable specificity, and a short detection time; thus, it can be regarded as an efficient technique for the rapid identification of pathogenic microorganisms in endophthalmitis. However, MALDI-TOF MS could not identify polymicrobial infection [43]. Being a highly selective analytical technique based on relative molecular mass, the mass-spectrometric component of MALDI-TOF MS provides 
an added advantage [40]. For the MALDI-TOF MS process, a matrix with energy-absorbing capacity is used to coat the samples. Exposure of the matrix-coated samples to a laser beam causes desorption and ionization of microbial analytes present in the sample. Ions from the sample move upward into a time-of-flight chamber based on their mass-to-charge ratio $(\mathrm{m} / \mathrm{z})$. Charge is detected and recorded using the charge detector at the top of the time-of-flight chamber; and this charge is unique for each analyte [44-46].

Using a laser pulse, MALDI-TOF MS works based on the rapid ionization of the bacterial ribosomal proteins directly from cultured colonies or cell pellets from the clinical sample (Figure 1). Bacterial colonies treated with ethanol-formic acid, as well as pure bacterial colonies, have been used for identification $[47,48]$. The direct smearing method of sample to prepare for MALDI-TOF MS analysis demonstrated higher accuracy of identification (99.85\%) compared with bacterial extraction pretreatment $(99.73 \%)$ when identifying
Acinetobacter species. Any incorrect identification was attributed to the absence of reference mass spectra in the MALDI-TOF MS database rather than sample preparation procedures [48]. In a study to access the ability of MALDITOF MS (Bruker Biotyper) to identify Acinetobacter species accurately in 286 blood isolates belonging to ACB complex and 39 other Acinetobacter spp., $85.3 \%$ of ACB complex and non-baumannii members were correctly identified by MALDITOF MS [49]. MALDI-TOF MS (Bruker Biotyper) correctly identified A. baumannii (98.6\%), A. nosocomialis (72.4\%), and $A$. pittii (97.6\%). Moreover, MALDI-TOF MS Bruker Biotyper correctly identified all A. ursingii, A. radioresistens, A. junnii, and A. jonhsonii isolates [49].

Jeong et al. [50] evaluated the ability of MALDI-TOF MS in combination with an improved database to identify various Acinetobacter species. They showed that the addition of 63 profiles for Acinetobacter strains to the default Bruker database increased the overall concordance rate between

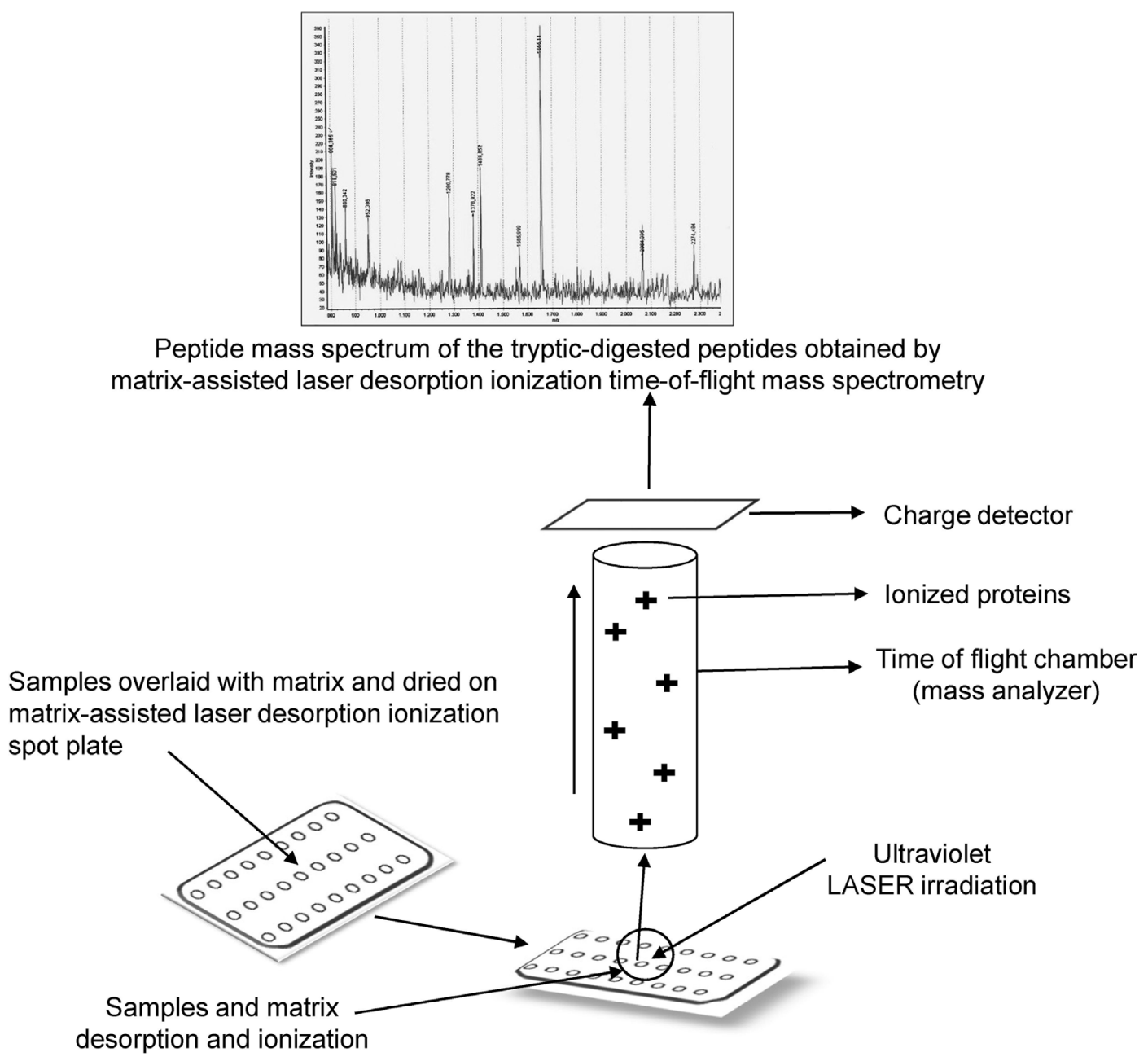

Figure 1. Diagrammatic representation of matrix-assisted laser desorption-ionization time-of-flight mass spectrometry work flow for microbial identification. 
MALDI-TOF MS and rpoB sequencing from $69.8 \%$ to $100.0 \%$. Additionally, after library modification, all 64 previously mismatched Acinetobacter strains were correctly identified. Sedo et al. [51] achieved accurate identification of Acinetobacter spp. by modification of the MALDI-TOF MS standard sample preparation procedure. The authors replaced $\alpha$-cyano-4-hydroxycinnamic acid (saturated solution in water :acetonitrile:trifluoroacetic acid, 47.5:50:2.5, v/v) with ferulic acid $(12.5 \mathrm{mg} / \mathrm{mL}$ solution in water:acetonitrile:formic acid 50:33:17, v/v), while other procedures remained unchanged, yielding more accurate identification and differentiation of A. baumannii and A. nosocomialis. However, a limitation is the automatic acquisition of mass spectra [51]. Toh et al. [52] analyzed the mass spectra of 73 Acinetobacter species, representing 10 different species, using an $\mathrm{AB}$ SCIEX 580 MALDI-TOF MS to differentiate members of the Acinetobacter genus, including the species of the ACB complex. They found that $A$. pittii or $A$. calcoaceticus, which could not be differentiated using $16 \mathrm{~S}$ rRNA and $r p o B$ gene sequencing, can be differentiated using gyr B multiplex PCR and MALDI-TOF MS [52].

Evidence from another study comparing MALDI-TOF $\mathrm{MS}$ and rpoB sequencing of 123 Acinetobacter spp. isolated from blood showed $86.2 \%$ (106/123) correct identification to species level and $13 \%$ identification to genus level by MALDITOF MS. Although the rpoB sequence analysis was correctly confirmed in $84 \%$ (89) of the 106 isolates identified by MALDITOF MS as correct, $16 \%$ of isolates were incorrectly identified. The authors recommended adding reference spectra to the MALDI-TOF MS database to improve identification capability [53]. This was affirmed when a study in which a database updated to include reference signatures for A. nosocomialis, A. seifertii, and A. dijkshoorniae, permitted correct speciation of 78 isolates. The correctly identified isolates included A. pittii, A. baumannii, A. dijkshoorniae, A. nosocomialis, and A. seifertii, indicating that regular updating of the MALDI-TOF MS databases is pivotal to accurate identification [47]. Many studies emphasize the advantages of MALDI-TOF MS as a promising tool to identify species rapidly [54]. A comparison of the dendrogram function of MALDI-TOF MS with pulsedfield gel electrophoresis demonstrated that MALDI-TOF MS dendrograms have insufficient discriminatory power for multidrug-resistant A. baumannii clonality analysis [54]. An earlier study, in agreement with the findings described above, found $100 \%$ identification and differentiation of A. baumannii, A. nosocomialis, A. pittii, A. calcoaceticus, A. dijkshoorniae, and $A$. seifertii when chemometric tools were incorporated into the MALDI-TOF MS [55]. This combined approach used a partial least squares discriminant analysis and hierarchical cluster analysis to obtain $100 \%$ correct species identification based on mass spectra generated from intact cells. Sousa et al. [55] found that $A$. dijkshoorniae and A. seifertii, neither of which were captured in the MALDI-TOF MS Biotyper database, were successfully differentiated.

Successful identification of all $18(100 \%)$ A. baumannii and $17(94 \%)$ of $18 \mathrm{~A}$. pittii isolates was achieved using the MALDI-TOF MS Bruker database to investigate 60 unrelated clinical isolates [56]. However, MALDI-TOF MS did not identify $A$. nosocomialis correctly until the signature profile for $A$. nosocomialis was included in the Bruker database. Variations in the protein signatures of A. baumannii, A. pittii, and $A$. nosocomialis are sufficient for accurate discrimination of the 3 species, based on the analysis performed on individual spectral peaks of representative strains [56]. A Korean study performed a comparative evaluation of the MALDI-TOF MS-based VITEK MS system versus the VITEK 2 and MicroScan automated systems to identify Acinetobacter species from blood cultures [57]. They found that the VITEK MS system is better than the VITEK 2 and MicroScan systems for the identification of Acinetobacter isolates, with fewer misidentifications and better discrimination between the $A$. baumannii and non- $A$. baumannii isolates [58] demonstrating a rapid and reliable identification of the 3 species of the ACB complex pathogenic for humans, namely, A. baumannii, A. pittii, and A. nosocomialis. The SARAMIS database of the Vitek MS was enriched and combined with a mass spectrometer to improve the identification of species of the ACB complex. For each species, reference spectra were obtained, and then a so-called "SuperSpectrum" was created based on the selection of 40 specific masses. In a second step, reference spectra and SuperSpectra with 100 isolates identified by $r p o \mathrm{~B}$ gene sequencing were validated. The evolution of the MALDI-TOF MS technology to accommodate clinical applications might be in sight as more discoveries may arise concerning Acinetobacter species taxonomy.

\section{Future perspectives in the identification of Acinetobacter}

The criterion standard used for diagnosis of A. baumannii infection is the traditional culture-biochemical method, which usually takes $24-72 \mathrm{~h}$ of incubation to produce accurate results. Consequently, this technique cannot detect causative pathogens early, which is crucial for the proper use of antimicrobial agents. Recently, several molecular methods were developed to detect and identify A. baumannii. However, these methods are labor-intensive and time-consuming. Owing to the limitations posed by available phenotypic and genotypic 
methods for bacterial identification, continuous efforts are being committed to the discovery and commercialization of new technologies that will make identification more straightforward and dramatically reduce the time required to obtain results. Coupling of analytical techniques and tests with new technologies, such as microfluidic devices is gaining more attention to achieve these goals. Some of these new technologies also exploit the use of ultrasensitive readout mechanisms and single-cell analysis [59, 60]. Miniaturized microfluidic devices and nanotechnologies are also beginning to find application in the area of antibiotic resistance detection and management, requiring only small samples, and less cumbersome, more cost-effective instruments, which together provide impressive high throughput analysis of single cells. The principle of microfluidic-based culture methods for bacteria detection is the monitoring of bacterial growth in small volumes housed within partitioned systems [21].

Some available identification tests depend on spectroscopic readings obtained from cell growth within a culture at numbers or levels sufficient for such monitoring. However, recent advances in this area now make it possible to monitor single cells for such identification and other observations rather than studying a group of cells together. More findings based on this principle are emerging. For instance, a rapid antibiotic susceptibility test system has been reported [61]. This microfluidic agarose channel coupled agarose in a microfluidic culture chamber to track single cells using microscopy to obtain images of the cell under varied antibiotic culture conditions, thus providing details of how the cell reacted to different antibiotic concentrations. Similar studies have also reported for several clinical bacterial pathogens [62-64]. Being small, these technologies and their like can be conveniently incorporated into automated, portable devices for use at points of care at a reduced cost compared with already existing detection methods [60].

Developments in miniaturized microfluidic detection systems are also advancing. These systems can significantly aid the automation of detection. An inertial microfluidic system was able to detect directly and separate bacteria pathogen in whole blood for direct identification by ribosomal RNA detection even when few pathogens (about $100 / \mathrm{mL}$ ) are present [65]. The system does not require culturing of the cells or any form of enzymatic amplification. Coupling microfluidic cell separation with RNA-base detection, the system can shorten detection and antibiotic susceptibility test to approximately $8 \mathrm{~h}$, representing a significant improvement in sample processing and therapeutic decision making. Another microfluidic system termed "Integrated Comprehensive Droplet Digital Detection" (IC 3D) with selective ability to detect single bacteria cells in a small quantity of blood without the need for culture or amplification has also been reported [66]. Similarly, Ismagilov et al. [67] reported a microfluidic system based on the separation of bacteria cells into nanovolume droplet plug for detection and screening for drug susceptibility.

New and more sensitive analytical methods with capacity for more rapid bacterial detection using mechanical and electrochemical transducers have been developed. Electrochemical sensors have been used in the identification of bacteria and resistance studies.

The developments mentioned above related to pathogen identification hold a promising future for Acinetobacter spp. differentiation, especially in clinical settings. Already, an optical biosensor has been developed, which used fluorescence to detect 57 pathogenic bacteria, including A. baumannii, in $10 \mathrm{~h}$. The detection limit was as low as $10 \mathrm{CFU} /$ assay and was successfully used to identify pathogens from blood specimens. A number of these technologies are still at the proof-of-concept stage, while some are still faced with the challenge of streamlining sample preparation, integration, and automation [60]. As these technologies continue to evolve and undergo improvements, they are likely to impact positively on available technologies for Acinetobacter spp. identification and differentiation, leading to improvements in patient care in cases arising from infections by clinically important members of the ACB complex.

\section{Conclusions}

Identification of Acinetobacter species among the phenotypically closely related ACB complex is fundamental for the administration of proper treatment. Significant advancement has been accomplished in the field of automated systems for bacterial identification, particularly for the A. baumannii complex leading to identification and differentiation of individual species among the members. The gains recorded through the use of MALDI-TOF MS has improved the process of identification of pathogens such as the ACB complex. Developments in microfluidic systems for pathogen detection appear to be advancing fast and hold great promise in reducing, if not solving, the ambiguity that exists with the differentiation of clinically important Acinetobacter spp.

Author contributions. $\mathrm{AB}$ contributed substantially to the conception, literature search, and draft of this review. GO, AH, and KS contributed substantially to the conception, design, and critical review of the manuscript. All authors approved the final version submitted for publication and take responsibility for statements made in the published article. 
Acknowledgments. The authors acknowledge support from the Universiti Sains Malaysia Bridging Grant 304. PPSP.6316142. Permanent addresses: Ahmad Ibrahim Bagudo, Department of Microbiology, Faculty of Life Science, Kebbi State University of Science and Technology, Aliero, Nigeria. Godwin Attah Obande, Department of Microbiology, Faculty of Science, Federal University, Lafia, Nasarawa State, Nigeria.

Conflicts of interest statement. The authors have each completed and submitted an International Committee of Medical Journal Editors Uniform Disclosure Form for Potential Conflicts of Interest. None of the authors has any potential of actual conflict of interest to disclose concerning the published article.

Data sharing statement. No new data sets were generated or analyzed during the present review. The present review is based on the references cited.

\section{References}

[1] de la Maza LM, Pezzlo MT, Bittencourt CE, Peterson EM. Acinetobacter, Chryseobacterium, Moraxella, Methylobacterium, and other nonfermentative Gram-negative bacilli. Color Atlas of Medical Bacteriology. 3rd ed. Washington, DC: ASM Press; 2020, p. 157-67.

[2] Vijayakumar S, Biswas I, Veeraraghavan B. Accurate identification of clinically important Acinetobacter spp.: an update. Future Sci OA. 2019; 5:FSO395. doi: 10.2144/fsoa-2018-0127

[3] Dijkshoorn L, Nemec A, Seifert H. An increasing threat in hospitals: multidrug-resistant Acinetobacter baumannii. Nat Rev Microbiol. 2007; 5:939-51.

[4] Clark NM, Zhanel GG, Lynch JP. Emergence of antimicrobial resistance among Acinetobacter species: a global threat. Curr Opin Crit Care. 2016; 22:491-9.

[5] Howard A, O'Donoghue M, Feeney A, Sleator RD. Acinetobacter baumannii. An emerging opportunistic pathogen. Virulence. 2012; 3:243-50.

[6] Gales AC, Seifert H, Gur D, Castanheira M, Jones RN, Sader HS. Antimicrobial susceptibility of Acinetobacter calcoaceticusAcinetobacter baumannii complex and Stenotrophomonas maltophilia clinical isolates: results from the SENTRY antimicrobial surveillance program (1997-2016). Open Forum Infect Dis. 2019; 6(Suppl 1): S34-46.

[7] Fitzpatrick MA, Ozer E, Bolon MK, Hauser AR. Influence of ACB complex genospecies on clinical outcomes in a U.S. hospital with high rates of multidrug resistance. J Infect. 2015; 70:144-52.

[8] Whistler T, Sangwichian O, Jorakate P, Sawatwong P, Surin U, Piralam B, et al. Identification of Gram negative non-fermentative bacteria: how hard can it be? PLoS Negl Trop Dis. 2019: 13:e0007729. doi: 10.1371/journal.pntd.0007729

[9] Gundi VAKB, Dijkshoorn L, Burignat S, Raoult D, La Scola B. Validation of partial $r p o B$ gene sequence analysis for the identification of clinically important and emerging Acinetobacter species. Microbiology (Reading). 2009; 155:2333-41.

[10] Higgins PG, Wisplinghoff H, Krut O, Seifert H. A PCR-based method to differentiate between Acinetobacter baumannii and Acinetobacter genomic species 13TU. Clin Microbiol Infect 2007; 13:1199-201.

[11] Higgins PG, Lehmann M, Wisplinghoff $H$, Seifert H. gyrB multiplex PCR to differentiate between Acinetobacter calcoaceticus and Acinetobacter genomic species 3. J Clin Microbiol. 2010; 48:4592-4.

[12] Šedo O, Nemec A, Kř́žová L, Kačalová M, Zdráhal Z. Improvement of MALDI-TOF MS profiling for the differentiation of species within the Acinetobacter calcoaceticus-Acinetobacter baumannii complex. Syst Appl Microbiol. 2013; 36:572-8.

[13] Cosgaya C, Marí-Almirall M, Van Assche A, Fernández-Orth D, Mosqueda N, Telli M, et al. Acinetobacter dijkshoorniae sp. nov., a member of the Acinetobacter calcoaceticus-Acinetobacter baumannii complex mainly recovered from clinical samples in different countries. Int J Syst Evol Microbiol. 2016; 66:4105-11.

[14] Nemec A, Krizova L, Maixnerova M, Sedo O, Brisse S, Higgins PG. Acinetobacter seifertii sp. nov., a member of the Acinetobacter calcoaceticus-Acinetobacter baumannii complex isolated from human clinical specimens. Int J Syst Evol Microbiol. 2015; 65:934-42

[15] Parida M, Sannarangaiah S, Dash PK, Rao PVL, Morita K. Loop mediated isothermal amplification (LAMP): a new generation of innovative gene amplification technique; perspectives in clinical diagnosis of infectious diseases. Rev Med Virol. 2008; 18:407-21.

[16] Ahmad I, Karah N, Nadeem A, Wai SN, Eric B, Uhlin BE. Analysis of colony phase variation switch in Acinetobacter baumannii clinical isolates. PLoS ONE. 2019; 14:e0210082. doi: 10.1371/journal. pone. 0210082

[17] Peleg AY, Seifert H, Paterson DL. Acinetobacter baumannii: emergence of a successful pathogen. Clin Microbiol Rev. 2008; 21:538-82.

[18] Bouvet PJM, Grimont PAD. Taxonomy of the genus Acinetobacter with the recognition of Acinetobacter baumannii sp. nov., Acinetobacter haemolyticus sp. nov., Acinetobacter johnsonii sp. nov., and Acinetobacter junii sp. nov. and emended descriptions of Acinetobacter calcoaceticus and Acinetobacter lwofii. Int J Syst Bacteriol. 1986; 36:228-40.

[19] Cerquetti M. Molecular typing in bacterial infections Haemophilus spp. In: de Filippis I, McKee M. editors. Molecular typing in bacterial infections. Totowa, NJ: Humana Press; 2013, p. 193-210.

[20] Maugeri G, Lychko I, Sobral R, Roque ACA. Identification and antibiotic-susceptibility profiling of infectious bacterial agents: a review of current and future trends. Biotechnol J. 2019; 14: 1700750. doi: 10.1002/biot.201700750

[21] Li Y, Yang X, Zhao W. Emerging microtechnologies and automated systems for rapid bacterial identification and antibiotic susceptibility testing. SLAS Technol. 2017; 22:585-608.

[22] Altheide ST. Biochemical and culture-based approaches to identification in the diagnostic microbiology laboratory. J Am Soc Clin Lab Sci. 2020. doi: 10.29074/ascls.119.001875

[23] Franco-Duarte R, Černáková L, Kadam S, Kaushik KS, Salehi B, Bevilacqua A, et al. Advances in chemical and biological methods to identify microorganisms-from past to present. 2019; Microorganisms. 2019; 7:130. doi: 10.3390/microorganisms7050130

[24] Váradi L, Luo JL, Hibbs DE, Perry JD, Anderson RJ, Orenga S, Groundwater PW. Methods for the detection and identification of 
pathogenic bacteria: past, present, and future. Chem Soc Rev. 2017; 46:4818-32.

[25] O'Hara CM. Manual and automated instrumentation for identification of Enterobacteriaceae and other aerobic gram-negative bacilli. 2005; 18:147-62.

[26] Bosshard PP, Zbinden R, Abels S, Bo B, Altwegg M, Bo EC. 16S rRNA gene sequencing versus the API 20 NE system and the VITEK 2 ID-GNB card for identification of nonfermenting gram-negative bacteria in the clinical laboratory. 2006; 44:1359-66.

[27] Stefanowicz A. The Biolog plates technique as a tool in ecological studies of microbial communities. Polish J Environ Stud. 2006; 15:669-76.

[28] Bernards AT, Dijkshoorn L, van der Toorn J, Bochner BR, van Boven CPA. Phenotypic characterisation of Acinetobacter strains of 13 DNA-DNA hybridisation groups by means of the Biolog system. J Med Microbiol. 1995; 42:113-9.

[29] Hernández-Durán M, López-Jácome LE, Colín-Castro CA, Cerón-González G, Ortega-Peña S, Vanegas-Rodríguez ES, et al. Comparison of the MicroScan WalkAway and VITEK 2 Compact systems for the identification and susceptibility of clinical Gram-positive and Gram-negative bacteria. Investigación en Discapacidad. 2017; 6:105-14.

[30] Snyder JW, Munier GK, Johnson CL. Direct comparison of the BD phoenix system with the MicroScan WalkAway system for identification and antimicrobial susceptibility testing of Enterobacteriaceae and nonfermentative gram-negative organisms. J Clin Microbiol. 2008; 46:2327-33.

[31] Kim S, Kim MH, Lee WI, Kang SY, Jeon YL. Misidentification of Acinetobacter baumannii as Alcaligenes faecalis by VITEK 2 system; case report. Lab Med. 2018; 49:e14-7.

[32] Benkova M, Soukup O, Marek J. Antimicrobial susceptibility testing: currently used methods and devices and the near future in clinical practice. J Appl Microbiol. 2020; 129:806-22.

[33] Wang J, Ruan Z, Feng Y, Fu Y, Jiang Y, Wang H, Yu Y. Species distribution of clinical Acinetobacter isolates revealed by different identification techniques. PLoS One. 2014; 9:e104882. doi:10.1371/ journal.pone.0104882

[34] Lee Y-C, Huang Y-T, Tan C-K, Kuo Y-W, Liao C-H, Lee P-I, Hsueh P-R. Acinetobacter baumannii and Acinetobacter genospecies 13TU and 3 bacteraemia: comparison of clinical features, prognostic factors and outcomes. J Antimicrob Chemother. 2011; 66:1839-46.

[35] Marschal M, Bachmaier J, Autenrieth I, Oberhettinger P, Willmann M, Petera S. Evaluation of the Accelerate Pheno system for fast identification and antimicrobial susceptibility testing from positive blood cultures in bloodstream infections caused by Gram-negative pathogens. J Clin Microbiol. 2017; 55:2116-26.

[36] Charnot-Katsikas A, Tesic V, Love N, Hill B, Bethel C, Boonlayangoor S, Bevis KG. Use of the Accelerate Pheno system for identification and antimicrobial susceptibility testing of pathogens in positive blood cultures and impact on time to results and workflow. J Clin Microbiol. 2018; 56:e01166-17. doi: 10.1128/ JCM.01166-17

[37] Ribeiro DG, Carmo LST, Santos IR, Almeida RF, Silva LP, Oliveira-Neto OB, et al. MALDI TOF MS-profiling: applications for bacterial and plant sample differentiation and biological variability assessment. J Proteomics. 2020;213:103619. doi: 10.1016/j. jprot.2019.103619

[38] Sogawa K, Watanabe M, Sato K, Segawa S, Ishii C, Miyabe A, et al. Use of the MALDI BioTyper system with MALDI-TOF mass spectrometry for rapid identification of microorganisms. Anal Bioanal Chem. 2011; 400:1905-11.

[39] Ge M-C, Kuo A-J, Liu K-L, Wen Y-H, Chia J-H, Chang P-Y, et al. Routine identification of microorganisms by matrix-assisted laser desorption ionization time-of-flight mass spectrometry: success rate, economic analysis, and clinical outcome. J Microbiol Immunol Infect. 2017; 50:662-8.

[40] Yan W, Qian J, Ge Y, Ye K, Zhou C, Zhang H. Principal component analysis of MALDI-TOF MS of whole-cell foodborne pathogenic bacteria. Anal Biochem. 2020; 592:113582. doi: 10.1016/j. ab.2020.113582

[41] Bar-Meir M, Berliner E, Kashat L, Zeevi DA, Assous MV. The utility of MALDI-TOF MS for outbreak investigation in the neonatal intensive care unit. Eur J Pediatr. 2020. doi: 10.1007/s00431-02003696-3.

[42] Yuan Y, Wang J, Zhang J, Ma B, Gao S, Li Y, et al. Evaluation of an optimized method to directly identify bacteria from positive blood cultures using MALDI-TOF mass spectrometry. J Clin Lab Anal. 2020; 34:e23119. doi: 10.1002/jcla.23119

[43] Xu S, Zhou C, Zhang P, Feng C, Zhang T, Sun Z, et al. Diagnostic performance of MALDI-TOF MS compared to conventional microbiological cultures in patients with suspected endophthalmitis. Ocul Immunol Inflamm. 2020; 28:483-90.

[44] Angeletti S. Matrix assisted laser desorption time of flight mass spectrometry (MALDI-TOF MS) in clinical microbiology. J Microbiol Methods. 2017; 138:20-9.

[45] Feucherolles M, Poppert S, Utzinger J, Becker SL. MALDI-TOF mass spectrometry as a diagnostic tool in human and veterinary helminthology: a systematic review. Parasit Vectors. 2019; 12:245. doi: 10.1186/s13071-019-3493-9

[46] Singhal N, Kumar M, Kanaujia PK, Virdi JS. MALDI-TOF mass spectrometry: an emerging technology for microbial identification and diagnosis. Front Microbiol. 2015; 6:791. doi: 10.3389/ fmicb.2015.00791

[47] Marí-Almirall M, Cosgaya C, Higgins PG, Van Assche A, Telli M, Huys G, et al. MALDI-TOF/MS identification of species from the Acinetobacter baumannii (Ab) group revisited: inclusion of the novel A. seifertii and A. dijkshoorniae species. Clin Microbiol Infect. 2017; 23:210.e1-9. doi: 10.1016/j.cmi.2016.11.020

[48] Li X, Tang Y, Lu X. Insight into identification of Acinetobacter species by matrix-assisted laser desorption/ionization time of flight mass spectrometry (MALDI-TOF MS) in the clinical laboratory. J Am Soc Mass Spectrom. 2018; 29:1546-53.

[49] Hsueh P-R, Kuo L-C, Chang T-C, Lee T-F, Teng S-H, Chuang Y-C, et al. Evaluation of the Bruker Biotyper matrix-assisted laser desorption ionization-time of flight mass spectrometry system for identification of blood isolates of Acinetobacter species. 2014; 52:3095-100.

[50] Jeong S, Hong JS, Kim JO, Kim KH, Lee W, Bae IK, et al. Identification of Acinetobacter species using matrix-assisted laser desorption ionization-time of flight mass spectrometry. Ann Lab Med. 2016; 36:325-34.

[51] Šedo O, Radolfová-Křížová L, Nemec A, Zdráhal Z. Limitations of routine MALDI-TOF mass spectrometric identification of Acinetobacter species and remedial actions. J Microbiol Methods. 2018; 154:79-85.

[52] Toh BEW, Paterson DL, Kamolvit W, Zowawi H, Kvaskoff D, Sidjabat H, et al. Species identification within Acinetobacter 


\section{Allli ASIAN BIOMEDICINE}

calcoaceticus-baumannii complex using MALDI-TOF MS. J Microbiol Methods. 2015; 118:128-32.

[53] Kishii K, Kikuchi K, Matsuda N, Yoshida A, Okuzumi K, Uetera Y, et al. Evaluation of matrix-assisted laser desorption ionizationtime of flight mass spectrometry for species identification of Acinetobacter strains isolated from blood cultures. Clin Microbiol Infect. 2014; 20:424-30.

[54] Rim JH, Lee Y, Hong SK, Park Y, Kim M, D’Souza R, et al. Insufficient discriminatory power of matrix-assisted laser desorption ionization time-of-flight mass spectrometry dendrograms to determine the clonality of multi-drug-resistant Acinetobacter baumannii isolates from an intensive care unit. Biomed Res Int. 2015; 2015:535027. doi: 10.1155/2015/535027

[55] Sousa C, Botelho J, Silva L, Grosso F, Nemec A, Lopes J, Peixe L. MALDI-TOF MS and chemometric based identification of the Acinetobacter calcoaceticus-Acinetobacter baumannii complex species. Int J Med Microbiol. 2014; 304:669-77.

[56] Espinal P, Martí S, Vila J. Effect of biofilm formation on the survival of Acinetobacter baumannii on dry surfaces. J Hosp Infect. 2012; 80:56-60.

[57] Lee W, Kim M, Yong D, Jeong SH, Lee K, Chong Y. Evaluation of VITEK mass spectrometry (MS), a matrix-assisted laser desorption ionization time-of-flight MS system for identification of anaerobic bacteria. Ann Lab Med. 2015; 35:69-75.

[58] Pailhoriès H, Daure S, Eveillard M, Joly-Guillou M-L, Kempf M. Using Vitek MALDI-TOF mass spectrometry to identify species belonging to the Acinetobacter calcoaceticus-Acinetobacter baumannii complex: a relevant alternative to molecular biology? Diagn Microbiol Infect Dis. 2015; 83:99-104.
[59] Gorgannezhad L, Stratton H, Nguyen NT. Microfluidic-based nucleic acid amplification systems in microbiology. Micromachines (Basel). 2019; 10:408. doi: 10.3390/mi10060408

[60] Kelley SO. New technologies for rapid bacterial identification and antibiotic resistance profiling. SLAS Technol. 2017; 22:113-21.

[61] Choi J, Jung Y-G, Kim J, Kim S, Jung Y, Na H, Kwon S. Rapid antibiotic susceptibility testing by tracking single cell growth in a microfluidic agarose channel system. Lab Chip. 2013; 13:280-7.

[62] Baltekin Ö, Boucharin A, Tano E, Andersson DI, Elf J. Antibiotic susceptibility testing in less than $30 \mathrm{~min}$ using direct single-cell imaging. Proc Natl Acad Sci U S A. 2017; 114:9170-5.

[63] Kara V, Duan C, Gupta K, Kurosawa S, Stearns-Kurosawa DJ, Ekinci KL. Microfluidic detection of movements of Escherichia coli for rapid antibiotic susceptibility testing. Lab Chip. 2018; 18:743-53.

[64] Malmberg C, Yuen P, Spaak J, Cars O, Tängdén T, Lagerbäck P. A novel microfluidic assay for rapid phenotypic antibiotic susceptibility testing of bacteria detected in clinical blood cultures. PLoS One. 2016; 11:e0167356. doi: 10.1371/journal.pone.0167356

[65] Hou HW, Bhattacharyya RP, Hung DT, Han J. Direct detection and drug-resistance profiling of bacteremias using inertial microfluidics. Lab Chip. 2015; 15:2297-307.

[66] Kang D-K, Ali MM, Zhang K, Huang SS, Peterson E, Digman MA, et al. Rapid detection of single bacteria in unprocessed blood using integrated comprehensive droplet digital detection. Nat Commun. 2014; 5:5427. doi: $10.1038 /$ ncomms6427

[67] Boedicker JQ, Li L, Kline TR, Ismagilov RF. Detecting bacteria and determining their susceptibility to antibiotics by stochastic confinement in nanoliter droplets using plug-based microfluidics. Lab Chip. 2008; 8:1265-72. 